\title{
Hepatic Resection After Prehepatectomy Chemotherapy for Metastatic Colorectal Cancer: A Propensity-Matched Analysis
}

\author{
DAISUKE KAWAGUCHI ${ }^{1}$, YUKIHIKO HIROSHIMA ${ }^{1}$, KENICHI MATSUO ${ }^{1}$, \\ ITARU ENDO ${ }^{2}$, KEIJI KODA ${ }^{1}$ and KUNIYA TANAKA ${ }^{1}$ \\ ${ }^{1}$ Department of Surgery, Teikyo University Chiba Medical Center, Chiba, Japan; \\ ${ }^{2}$ Department of Gastroenterological Surgery, Yokohama City University \\ Graduate School of Medicine, Yokohama, Japan
}

\begin{abstract}
Background: Liver metastases from colorectal cancer (CRC) are best managed using multiple modalities, but the role of chemotherapy prior to resection of marginally resectable liver metastases remains unsettled. Patients and Methods: Consecutive patients treated for marginally resectable CRC liver metastases were matched using a propensity-score analysis based on the probability of a patient having up-front surgery or prehepatectomy chemotherapy followed by surgery. Results: The study group consisted of 70 propensity-matched patients undergoing up-front surgery or prehepatectomy chemotherapy followed by surgery. Groups were similar in terms of baseline characteristics. Median estimated blood loss (605 $\mathrm{ml} \mathrm{vs.} 957 \mathrm{ml}, p=0.006)$, number of patients requiring transfusion ( $5 v s .14, p=0.016)$ and median postoperative hospital stay (13 vs. 17 days, $p=0.005$ ) were significantly less in the prehepatectomy chemotherapy group. Conclusion: Hepatic resection after prehepatectomy chemotherapy for patients with marginally resectable CRC liver metastases can lead to favorable short-term outcomes.
\end{abstract}

The liver is the most common site of metastasis in patients with colorectal cancer (CRC). Liver resection is the standard-of-care for patients with metastatic colorectal cancer confined to the liver. Over the past decade, major advances in chemotherapy have substantially improved chances of cure for patients with advanced liver metastases from CRC. With advances in liver surgery, perioperative care, and chemotherapy, the traditional clinical criteria for

Correspondence to: Kuniya Tanaka, MD, Department of Surgery, Teikyo University Chiba Medical Center, 3426-3 Anesaki, Ichihara, Chiba, 299-0111, Japan. Tel: +81 436621211, Fax: +81 436613961, e-mail: U17-92TS@med.teikyo-u.ac.jp

Key Words: Metastatic colorectal cancer, prehepatectomy chemotherapy. resection -based on number, size, or location of liver metastases - no longer limit opportunities for curative resection (1). At present, any patient in whom metastatic disease can be cleared with negative margins while still maintaining an adequate remnant liver should be considered for resection. Nonetheless, patients who undergo successful hepatic resection have a high risk of developing recurrent disease, either in the liver or elsewhere $(2,3)$. In order to reduce this risk, a multimodal approach using systemic chemotherapy in combination with resection has evolved as an important strategy for treatment of metastatic CRC. Progress in both surgery and chemotherapy have resulted in marked improvements in overall survival (OS), with recent single- and multicenter studies reporting 5-year survival rates of approximately $50 \%$ after liver resection for metastatic CRC (2-7).

Advances in prehepatectomy chemotherapy, including more effective chemotherapeutic and biological agents, now permit novel strategies for treating resectable and initially unresectable CRC liver metastases (8). However, when metastatic disease is marginally resectable, deferring surgery until after chemotherapy remains controversial. Theoretical advantages of prehepatectomy chemotherapy include the potential of reducing the extent of resection, increasing the $\mathrm{R} 0$ resection rate, eradicating micrometastatic disease, and offering opportunity for assessment of individual patient response to specific chemotherapy regimens. Disadvantages of prehepatectomy chemotherapy include the risk of tumor progression to an unresectable state, chemotherapyassociated hepatotoxicity leading to increased perioperative morbidity, and the possibility of chemotherapy-responsive but still-viable tumors shrinking to an undetectable size, rendering subsequent localization and resection more difficult (9-12). These opposing risks and benefits have occasioned ongoing debate regarding timing of chemotherapy for patients with marginally resectable liver metastases. 
This preliminary study is a propensity score-matched analysis of short- and long-term outcomes in patients with marginally resectable liver metastases who undergo hepatectomy after chemotherapy compared to those in patients undergoing up-front surgery.

\section{Patients and Methods}

Patients. Patients with histologically confirmed CRC who developed liver metastases were eligible. Patients receiving adjuvant chemotherapy for their primary tumor within 6 months before resection of their liver metastases were excluded. To be included, patients had to meet at least one of the following criteria for marginal resectability of hepatic metastases, as assessed by a local multidisciplinary team: at least four liver metastases, with involvement of both major lobes; metastases at least $80 \mathrm{~mm}$ in diameter and unfavorably located (invasion of major vessels); and presence of concomitant extrahepatic metastases according to traditional criteria of unresectability (13). Unresectable metastases that could not be cleared while maintaining an adequate remnant liver excluded a patient from this study.

Hepatic lesions were assessed using three-phase computed tomography $(\mathrm{CT})$ or magnetic resonance imaging (MRI) including liver-specific contrast agent.

One hundred and forty-five consecutive patients who recently underwent liver resection for marginally resectable liver metastases from CRC and met the above inclusion criteria were included in this analysis. Among these, 81 patients received prehepatectomy chemotherapy and 64 patients underwent up-front surgery. For each patient, the strategy used, chemotherapy-first or surgery-first, was decided on a case-by-case basis. Most chemotherapy-first patients were referred from other institutions after undergoing chemotherapy. Therefore, patients were not randomly allocated to undergo chemotherapy or up-front surgery. In order to control for selection bias, a propensity score was devised and used as an adjustment variable. Patients who received chemotherapy first were matched at a 1:1 ratio with patients who underwent up-front surgery on a nearest-neighbor basis, based on age, gender, number of metastases, maximum size of metastases, primary site, distribution, and presence or absence of extrahepatic metastases. Oncological outcomes were compared between the two groups.

Perihepatectomy chemotherapy. In prehepatectomy chemotherapy, the components of regimens, numbers of lines used for treatment administration, and numbers of courses given usually depended upon individual patient responses to chemotherapy, or upon oncologists' opinions. Regimens were usually combinations of 5fluorouracil (5-FU), 1-folinic acid (FA), and oxaliplatin with/without irinotecan, with or without use of a monoclonal antibody. Response to chemotherapy was evaluated using CT, according to the Response Evaluation Criteria in Solid Tumors criteria (RECIST) criteria (14).

Hepatectomy procedures and patient safety assesment. In order to determine whether or not hepatectomy was acceptably safe for a patient, we used a predictive score (PS) introduced by Yamanaka et al. (15). The PS was calculated using the formula PS $=-84.6+0.933$ $a+1.11 b+0.999 c$, with ' $a$ ' representing the anticipated resection fraction $(\%)$ calculated by volumetry using computed tomography $(\mathrm{CT})$; ' $b$ ', indocyanine green retention rate at $15 \mathrm{~min}(\%)$; and ' $c$ ', patient age in years. A PS less than 50 indicated that a given hepatectomy would be acceptable. Disease in patients with a PS of 50 or more was considered unresectable; these patients were treated with a two-stage approach with or without prehepatectomy portal vein embolization after administration of conversion chemotherapy. A major hepatectomy was defined as a resection of at least two sections according to the Brisbane 2000 terminology of the International Hepato-Pancreato-Biliary Association (16).

Microscopic evaluation. Histopathological findings in resected nontumor liver tissue were evaluated semi-quantitatively as follows. The degree of steatosis was graded as none, mild $(<30 \%$ of hepatocytes), moderate $(\geq 30 \%$ to $50 \%)$ or severe $(\geq 50 \%)$; steatohepatitis was graded as defined by Kleiner et al. (17) based on steatosis (score $0,<5 \% ; 1,5 \%$ to $33 \% ; 2,>33 \%$ to $66 \%$; and 3 , $>66 \%$ ), lobular inflammation (score 0 , no foci; 1 , one focus per 200 $\times$ field; 2,2 to 4 foci; and 3,>4 foci); and hepatocytic ballooning (score 0 , none; 1 , few balloon cells; and 2 , many cells/prominent ballooning). Sinusoidal injury was graded according to an established grading system based on sinusoidal dilation (grade 0 , absent; grade 1, centrilobular involvement limited to one-third of the lobule; grade 2, centrilobular involvement extending to involve two-thirds of the lobule; and grade 3, complete lobular involvement) (18). Liver injury was defined as steatosis of at least $30 \%$, Kleiner steatohepatitis score of at least 4 , with/without sinusoidal dilation of at least grade 2 to 3 .

Adjuvant chemotherapy. After resection for liver metastases or extrahepatic metastases, patients generally received additional adjuvant chemotherapy, generally with 5-FU and FA with or without oxaliplatin, or irinotecan.

Patient follow-up. One week after liver resection, CT was performed to confirm liver regeneration status and to check for any intraabdominal abnormality related to the operation. Assessment of complications followed a recently published standardized complication classification system (Clavien-Dindo classification) (19). Complications were defined as any deviation from an uneventful postoperative course within 30 days of operation. Posthepatectomy liver failure (PHLF) was diagnosed based on the International Study Group of Liver Surgery (ISGLS) definition (20). In brief, the ISGLS definition of PHLF requires increased international normalized ratio and hyperbilirubinemia on or after postoperative day 5. PHLF was categorized further using three grades of severity: grade A, PHLF resulting in abnormal laboratory parameters but requiring no change in clinical management of the patient; grade B, PHLF resulting in a deviation from regular clinical management but manageable without invasive treatment; and grade C, PHLF resulting in a deviation from regular clinical management and requiring invasive treatment. Patients underwent follow-up evaluation monthly at an outpatient clinic. Serum carcinoembryonic antigen (CEA), carbohydrate antigen (CA) 19-9, and p53 were measured every month; CT was performed every 3 to 4 months for 5 years after the most recent operation.

Statistical analysis. Statistical comparisons of baseline data were performed by the Mann-Whitney $U$-test, the $\chi^{2}$ test, or Fisher's exact test as appropriate. Survival rates were calculated by the Kaplan-Meier method. A difference was considered significant when the two-sided $p$-value was below 0.05 . 
Table I. Overall patient and profiles for the two treatment groups.

\begin{tabular}{|c|c|c|c|}
\hline Variable & $\begin{array}{l}\text { Up-front } \\
\text { surgery } \\
(n=35)\end{array}$ & $\begin{array}{l}\text { Prehepatectomy } \\
\text { chemotherapy } \\
(n=35)\end{array}$ & $p$-Value \\
\hline \multicolumn{4}{|l|}{ Patient-related } \\
\hline Age, years & $62(62-78)$ & $65(41-74)$ & 0.370 \\
\hline \multicolumn{4}{|l|}{ Gender } \\
\hline Male & $26(74 \%)$ & $27(77 \%)$ & \multirow[t]{2}{*}{0.780} \\
\hline Female & $9(26 \%)$ & $8(23 \%)$ & \\
\hline \multicolumn{4}{|l|}{ Tumor-related } \\
\hline \multicolumn{4}{|l|}{ Distribution } \\
\hline Unilobar & $7(20 \%)$ & $2(6 \%)$ & \multirow[t]{2}{*}{0.074} \\
\hline Bilobar & $28(80 \%)$ & $33(94 \%)$ & \\
\hline Number & $4(4-19)$ & $5(6-22)$ & 0.547 \\
\hline Maximum tumor size, $\mathrm{mm}$ & $45(24-120)$ & $42(20-150)$ & 0.622 \\
\hline \multicolumn{4}{|l|}{ Primary site } \\
\hline Colon & $16(46 \%)$ & $20(57 \%)$ & \multirow[t]{2}{*}{0.339} \\
\hline Rectum & $19(54 \%)$ & $15(43 \%)$ & \\
\hline \multicolumn{4}{|l|}{ Extrahepatic metastasis } \\
\hline Present & $8(23 \%)$ & $7(20 \%)$ & \multirow[t]{2}{*}{0.771} \\
\hline \multirow[t]{2}{*}{ Serum CEA, ng/ml } & 125.1 & 193.2 & \\
\hline & $(5.7-880)$ & $(7.5-560)$ & 0.641 \\
\hline \multicolumn{4}{|l|}{ Treatment-related } \\
\hline \multicolumn{4}{|l|}{ Prehepatectomy CTx } \\
\hline Performed & $0(0 \%)$ & $35(100 \%)$ & 0.001 \\
\hline \multicolumn{4}{|l|}{ Portal vein embolization } \\
\hline Performed & $27(77 \%)$ & $22(63 \%)$ & 0.192 \\
\hline \multicolumn{4}{|l|}{ Staged hepatectomy } \\
\hline Performed & $27(77 \%)$ & $22(63 \%)$ & 0.192 \\
\hline \multicolumn{4}{|l|}{ Extent of hepatectomy } \\
\hline Major & $21(60 \%)$ & $20(57 \%)$ & 0.808 \\
\hline \multicolumn{4}{|l|}{ Curability of liver metastasis } \\
\hline $\mathrm{R} 0 / \mathrm{R} 1$ & $29(83 \%)$ & $32(91 \%)$ & 0.284 \\
\hline $\mathrm{R} 2$ & $6(17 \%)$ & $3(9 \%)$ & \\
\hline
\end{tabular}

Values of continuous variables are medians. Ranges are shown in parentheses. CEA, Carcinoembryonic antigen, CTx, chemotherapy.

\section{Results}

After application of the propensity score-matching procedure, the study group was restricted to 35 propensitymatched pairs (70 patients) undergoing prehepatectomy chemotherapy and up-front surgery, respectively. In addition to the matched variables (age, gender, number of metastases, maximum size, primary site, distribution, and presence or absence of extrahepatic metastases), no differences of other variables were observed between groups, except regarding prehepatectomy chemotherapy (Table I). Four patients received six cycles of prehepatectomy chemotherapy via the hepatic artery with a combination of 5-FU, FA, and cisplatin. Eleven patients both received three cycles of hepatic arterial infusion chemotherapy, as outlined above and three cycles of systemic chemotherapy. The other 20 patients received only systemic chemotherapy. Regimens used for systemic chemotherapy consisted of FOLFOX (5-FU and FA with
Table II. Perioperative variables and morbidity

\begin{tabular}{lccc}
\hline Variable & $\begin{array}{c}\text { Up-front } \\
\text { surgery } \\
(\mathrm{n}=35)\end{array}$ & $\begin{array}{c}\text { Prehepatectomy } \\
\text { chemotherapy } \\
(\mathrm{n}=35)\end{array}$ & $p$-Value \\
\hline Operative time, min & $431.3 \pm 120.4$ & $418.2 \pm 113.8$ & 0.650 \\
& $(431,222-780)$ & $(406,245-736)$ & \\
Blood loss, ml & $1145.3 \pm 644.1$ & $778.2 \pm 454.7$ & 0.006 \\
Transfused patients & $(957,300-2500)$ & $(605,310-1700)$ & \\
Morbidity & $14(40 \%)$ & $5(14.2 \%)$ & 0.016 \\
Bile leakage & $5(14.2 \%)$ & $4(11.4 \%)$ & 0.721 \\
Surgical site infection & 0 & $1(2.8)$ & 0.314 \\
Intra-abdominal abscess & $2(5.6 \%)$ & $1(2.8)$ & 0.314 \\
Liver failure & 0 & $2(5.6 \%)$ & 1.000 \\
Respirator-related infection & $3(8.4 \%)$ & $1(2.8 \%)$ & 0.314 \\
Hospital stay, days & $18.6 \pm 9.4$ & $13.5 \pm 4.4$ & 0.005 \\
& $(17,7-55)$ & $(13,7-27)$ & \\
\hline
\end{tabular}

Values of continuous variables are mean $\pm \mathrm{SD}$, followed by medians and ranges in parentheses.

oxaliplatin) in 12 patients and FOLFIRI (5-FU and FA with irinotecan) in six patients. Additionally, regimens included monoclonal antibodies in 13 patients: FOLFOX plus bevacizumab in eight patients, FOLFIRI plus bevacizumab in two patients, FOLFOX plus cetuximab in two patients, and FOLFIRI plus cetuximab in one patient. The median numbers of lines used for administration and of courses of prehepatectomy chemotherapy respectively were one (range=1-5) and 5 (range=2-16) in the prehepatectomy chemotherapy group. Adjuvant chemotherapy was performed in 22 patients $(63 \%)$ in the prehepatectomy chemotherapy group and 27 patients in the up-front surgery group (77\%, $p=0.192)$.

Perioperative variables and short-term outcome. Operative time did not differ significantly between the two groups. Intraoperative blood loss and incidence of blood transfusion were greater in the up-front surgery group than the prehepatectomy chemotherapy group (Table II). No mortality occurred in either group. Severity and incidence of postoperative complications were grade I in one patient $(2.8 \%)$, grade II in one $(2.8 \%)$, and grade IIIa in two $(5.6 \%)$ in the prehepatectomy chemotherapy group; these were grade II in four (11.4\%) and grade IIIa in one $(2.8 \%)$ case in the up-front surgery group. PHLF rates in the prehepatectomy chemotherapy and in the up-front surgery group were $2.8 \%$ (one patient, grade A) and $0 \%$, respectively. Hospital stay was longer in the up-front surgery group than in the prehepatectomy chemotherapy group $(p=0.005)$. Other details of complications in each group are listed in Table II. 
Long-term survival and freedom from recurrence. The median observation period in the up-front group was 3.94 years, and that in the neoadjuvant chemotherapy group were 3.29 years $(p=0.305)$. Overall survival, disease-free rates and remnant liver recurrence-free rates after final hepatectomy did not differ between groups (Figure 1). Responses to final chemotherapy in the neoadjuvant group were partial response (PR) in 17 patients, stable disease (SD) in 12, and progressive disease $(\mathrm{PD})$ in six. When patients who received prehepatectomy chemotherapy were stratified according to response to chemotherapy, no difference in overall survival was seen between patients with up-front surgery and patients with prehepatectomy chemotherapy whose response to chemotherapy represented PR, SD or PD ( $p=0.867,0.834$ and 0.400 , respectively). Disease-free rate and liver recurrence-free rate also were comparable between patients with up-front surgery and prehepatectomy chemotherapy patients with PR, SD or PD ( $p=0.906,0.613$ and 0.329 , and $p=0.678,0.434$ and 0.909 , respectively).

Recurrences and repeat hepatectomy. After liver resection, recurrences were detected in 25 patients (71\%) in the upfront surgery group (Table III). Recurrences were observed in 30 patients in the prehepatectomy chemotherapy group ( $86 \%, p=0.24 v s$. the up-front surgery group). Numbers and sites of initial recurrence, as well as numbers of repeat hepatectomies performed for liver recurrence, did not differ significantly between the two groups.

Histopathological evaluation of liver injury. Among patients who received neoadjuvant chemotherapy, steatosis exceeding $30 \%$ was observed in eight patients $(22.8 \%)$; grade 2 to 3 sinusoidal dilation in six patients $(17.1 \%)$; and Kleiner steatohepatitis score of 4 or more in five patients (14.3\%). Steatosis and sinusoidal dilation were greater, and steatohepatitis score tended to be greater in the prehepatectomy chemotherapy group than the up-front surgery group $(p=0.012,0.046$, and 0.232 ; Table IV).

\section{Discussion}

In this study, we compared early and long-term outcomes in a propensity score-matched series of patients with marginally resectable liver metatstases receiving either up-front surgery or chemotherapy followed by surgery. The role of chemotherapy prior to resection of marginally resectable liver metatstases from CRC is considered uncertain.

Histological changes in the liver induced by chemotherapy, including steatohepatitis and vascular injury, may be associated with increased perioperative complications $(11,16)$. We found prehepatectomy chemotherapy to be significantly associated with greater likelihood of steatosis and sinusoidal dilation than up-front surgery $(22.8 \% v s .2 .8 \%$ for steatosis,
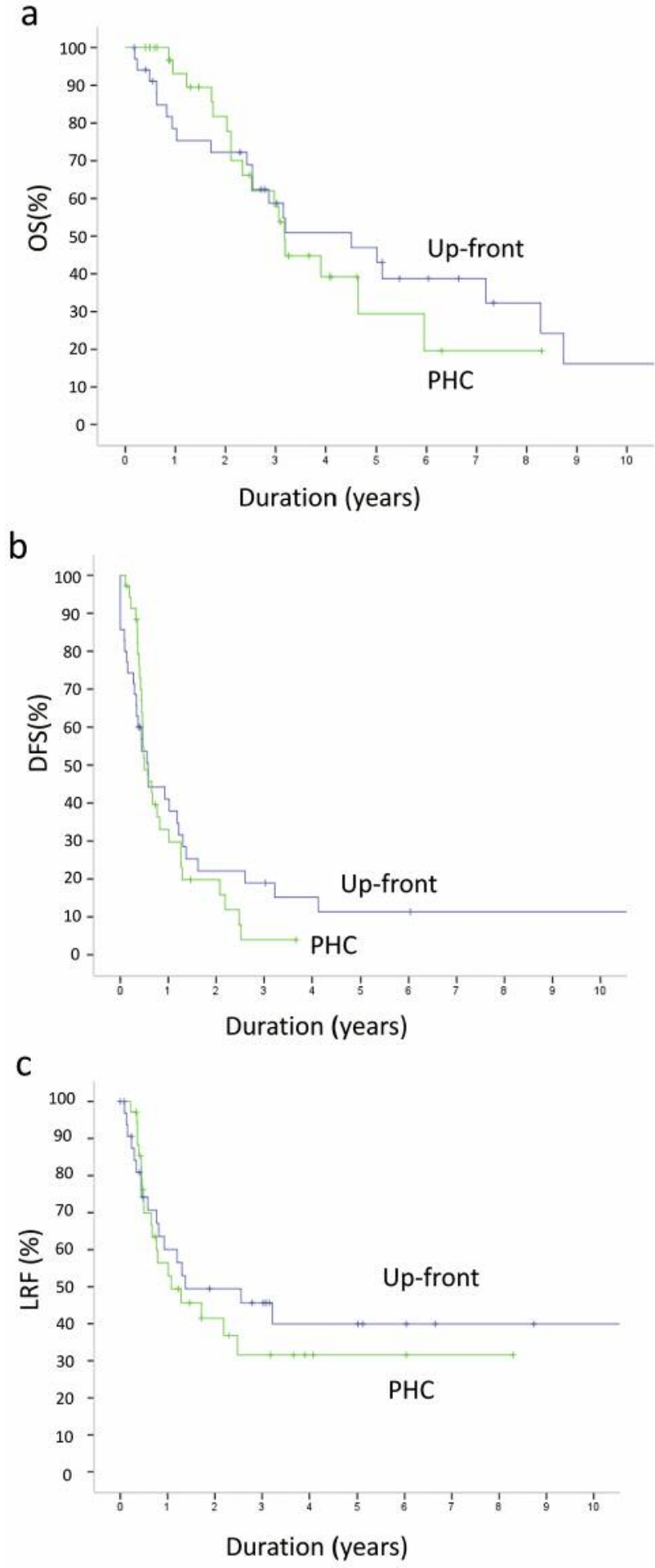

Figure 1. Cumulative overall survival (OS) (a), disease-free rate (DFS) (b) and liver recurrence-free $(L R F)(c)$ rates, after liver resection for patients with marginally resectable metastases in up-front surgery (Up-front; blue line, $n=35)$ vs. prehepatectomy chemotherapy (PHC; green line, $n=35)$. No significant differences for overall survival $(p=0.711)$, disease-free survival; panel $b, p=0.601)$, or recurrence-free rates $(p=0.576)$ were seen between up-front surgery and prehepatectomy chemotherapy groups. 
Table III. Details of recurrence after hepatectomy.

\begin{tabular}{lccc}
\hline Variable & $\begin{array}{c}\text { Up-front } \\
\text { surgery } \\
(\mathrm{n}=35)\end{array}$ & $\begin{array}{c}\text { Prehepatectomy } \\
\text { chemotherapy } \\
(\mathrm{n}=35)\end{array}$ & $p$-Value \\
& & & \\
\hline $\begin{array}{l}\text { Number of initial } \\
\text { recurrence sites }\end{array}$ & $21(60 \%)$ & $21(60 \%)$ & 0.133 \\
$\quad 1$ & $4(11 \%)$ & $9(26 \%)$ & \\
2 & $9(26 \%)$ & $6(17 \%)$ & 0.382 \\
Site of initial recurrence & $8(22 \%)$ & $8(22 \%)$ & 1.000 \\
$\quad$ Liver & $4(11 \%)$ & $7(20 \%)$ & 0.324 \\
$\quad$ Lung & $4(11 \%)$ & $9(26 \%)$ & 0.124 \\
$\quad \begin{array}{l}\text { Other site } \\
\text { Multiple }\end{array}$ & & & \\
Repeat hepatectomy & $6(17 \%)$ & $5(14 \%)$ & 0.743 \\
$\quad$ Performed & & & \\
\hline
\end{tabular}

Table IV. Liver injury characteristics.

\begin{tabular}{lccc}
\hline Liver injury & $\begin{array}{c}\text { Up-front } \\
\text { surgery } \\
(\mathrm{n}=35)\end{array}$ & $\begin{array}{c}\text { Prehepatectomy } \\
\text { chemotherapy } \\
(\mathrm{n}=35)\end{array}$ & $p$-Value \\
\hline Steatosis $>30 \%$ & $1(2.8 \%)$ & $8(23 \%)$ & 0.012 \\
Sinusoidal dilation & $1(2.8 \%)$ & $6(17 \%)$ & 0.046 \\
Steatohepatitis & $2(5.6 \%)$ & $5(14 \%)$ & 0.232 \\
\hline
\end{tabular}

$p=0.012$; and $17.1 \%$ vs. $2.8 \%$ for sinusoidal dilation, $p=0.046$ ). These findings are essentially in agreement with another recently published study (21). However, we experienced no mortality in either group, while morbidity rates also did not differ significantly between the two groups. According to a previous report (22), risk of morbidity and mortality may be related to differences in number of lines used for chemotherapy and to duration of treatment. One possible explanation for our avoidance of perioperative deaths in both groups and for the absence of significant differences in perioperative morbidity between groups is that our prehepatectomy chemotherapy patients had few lines and courses of chemotherapy (respective medians, 1 and 5).

Although no significant difference in extent of hepatectomy was observed between groups, patients undergoing prehepatectomy chemotherapy experienced less blood loss, fewer transfusions, and shorter hospital stays. We attribute this result to greater ease in surgically manipulating tumors in the prehepatectomy chemotherapy group as a result of more even, less invasive surface contours of tumors after prehepatectomy chemotherapy. The first operative priority during liver surgery should be to minimize bleeding, as perioperative hemorrhage has been associated with increased morbidity and mortality (23). Although we found no significant difference in morbidity between groups, we suspect that more blood loss and more transfusions contributed to longer hospital stays for up-front surgery patients.

We found similar DFS, OS and liver recurrence-free survival whether patients had up-front surgery or prehepatectomy chemotherapy. In a report of experience with resectable liver metastases, resection patients who received chemotherapy had a significant increase in DFS, with survival rate at 3 years increased by $9.2 \%$ (hazard ratio $=0.73, p=0.025$ ) (24). However, no significant improvement in OS was seen for patients with liver metatstases from CRC who received perioperative chemotherapy rather than surgery alone (25). Thus, while neoadjuvant chemotherapy for patients with resectable disease may improve progression-free survival, OS is not improved. In our study, prehepatectomy chemotherapy for patients with marginally resectable metastases did not show a significant improvement of long-term outcome.

Our present study results suggest that compared to up-front surgery, a chemotherapy-first strategy can be effective in reducing intraoperative blood loss, need for transfusion, and length of hospital stay in patients with marginally resectable metastatic disease, but not in significantly improving DFS, OS, or freedom from liver recurrence. Limitations of our study include the biases inherent in a retrospective review of unselected cases, the fact that not all patients received postoperative chemotherapy, and the fact that indications for chemotherapy and the choice of agents used were not standardized. One also might argue that a chemotherapy-first strategy delays resection, increasing the risk of tumor progression to an unresectable state if the patient does not respond to chemotherapy. However, delay sufficient to allow disease progression to an unresectable state in patients with marginally resectable metastatic disease would seem unlikely with administration of only a few cycles of chemotherapy. In the present study, a chemotherapy-first strategy showed favorable short-term outcomes, and refinements of treatment might allow for better long-term outcomes in the future. Randomized trials are needed to more definitively address timing of perioperative chemotherapy, and to prospectively collect data using validated stratification systems to guide the choice of treatment strategy. Even now, however, our shortterm results may favor chemotherapy prior to the resection of marginally resectable liver metatstases from CRC.

\section{Financial Disclosures}

The Authors have no financial disclosures to make in regard to this study.

\section{References}

1 Steele G Jr. and Ravikumar TS: Resection of hepatic metastases from colorectal cancer. Biologic perspective. Ann Surg 210: 127138, 1989. 
2 de Jong MC, Pulitano C, Ribero D, Strub J, Mentha G, Schulick RD, Choti MA, Aldrighetti L, Capussotti L and Pawlik TM: Rates and patterns of recurrence following curative intent surgery for colorectal liver metastasis: An international multiinstitutional analysis of 1669 patients. Ann Surg 250: 440-448, 2009.

3 Pawlik TM, Scoggins CR, Zorzi D, Abdalla EK, Andres A, Eng C, Curley SA, Loyer EM, Muratore A, Mentha G, Capussotti L and Vauthey JN: Effect of surgical margin status on survival and site of recurrence after hepatic resection for colorectal metastases. Ann Surg 241: 715-722, 2005.

4 House MG, Ito H, Gonen M, Fong Y, Allen PJ, DeMatteo RP, Brennan MF, Blumgart LH, Jarnagin WR and D'Angelica MI: Survival after hepatic resection for metastatic colorectal cancer: trends in outcomes for 1600 patients during two decades at a single institution. J Am Coll Surg 210: 744-755, 2010.

5 Figueras J, Torras J, Valls C, Llado L, Ramos E, Marti-Ragué J, Serrano T and Fabregat J: Surgical resection of colorectal liver metastases in patients with expanded indications: a single-center experience with 501 patients. Dis Colon Rectum 50: 478-488, 2007.

6 Abdalla EK, Vauthey JN, Ellis LM, Ellis V, Pollock R, Broglio $\mathrm{KR}, \mathrm{Hess} \mathrm{K}$ and Curley SA: Recurrence and outcomes following hepatic resection, radiofrequency ablation, and combined resection/ablation for colorectal liver metastases. Ann Surg 239: 818-825, 2004.

7 Wei AC, Greig PD, Grant D, Taylor B, Langer B and Gallinger S: Survival after hepatic resection for colorectal metastases: a 10-year experience. Ann Surg Oncol 13: 668-676, 2006.

8 Nordlinger B and Benoist S: Benefits and risks of neoadjuvant therapy for liver metastases. J Clin Oncol 24: 4954-4955, 2006.

9 Aloia T, Sebagh M, Plasse M, Karam V, Lévi F, Giacchetti S, Azoulay D, Bismuth $\mathrm{H}$, Castaing D and Adam R: Liver histology and surgical outcomes after preoperative chemotherapy with fluorouracil plus oxaliplatin in colorectal cancer liver metastases. J Clin Oncol 24: 4983-4990, 2006.

10 Karoui M, Penna C, Amin-Hashem M, Mitry E, Benoist S, Franc $\mathrm{B}$, Rougier $\mathrm{P}$ and Nordlinger B: Influence of preoperative chemotherapy on the risk of major hepatectomy for colorectal liver metastases. Ann Surg 243: 1-7, 2006.

11 Vauthey JN, Pawlik TM, Ribero D, Wu TT, Zorzi D, Hoff PM, Xiong HQ, Eng C, Lauwers GY, Mino-Kenudson M, Risio M, Muratore A, Capussotti L, Curley SA and Abdalla EK: Chemotherapy regimen predicts steatohepatitis and an increase in 90-day mortality after surgery for hepatic colorectal metastases. J Clin Oncol 24: 2065-2072, 2006.

12 Zalinski S, Abdalla EK, Mahvash A and Vauthey JN. A marking technique for intraoperative localization of small liver metastases before systemic chemotherapy. Ann Surg Oncol 16: 1208-1211, 2009.

13 Bismuth H, Adam R, Levi F, Farabos C, Waechter F, Castaing D, Majno P and Engerran L: Resection of nonresectable liver metastases from colorectal cancer after neoadjuvant chemotherapy. Ann Surg 224: 509-522, 1996.

14 Therasse P, Arbuck SG, Eisenhauer EA, Wanders J, Kaplan RS, Rubinstein L, Verweij J, Van Glabbeke M, van Oosterom AT, Christian MC and Gwyther SG: New guidelines to evaluate the response to treatment in solid tumors. J Natl Cancer Inst 92: 205-216, 2000.
15 Yamanaka N, Okamoto E, Oriyama T, Fujimoto J, Furukawa K, Kawamura E, Tanaka $\mathrm{T}$ and Tomoda F: A prediction scoring system to select the surgical treatment of liver cancer. further refinement based on 10 years of use. Ann Surg 219: 342-346, 1994.

16 Pang YY: The brisbane terminology of liver anatomy and resections. HPB (Oxford) 2: 333-339, 2000.

17 Kleiner DE, Brunt EM, Van Natta M, Behling C, Contos MJ, Cummings OW, Ferrell LD, Liu YC, Torbenson MS, UnalpArida A, Yeh M, McCullough AJ and Sanyal AJ: Design and validation of a histological scoring system for nonalcoholic fatty liver disease. Hepatology 41: 1313-1321, 2005.

18 Rubbia-Brandt L, Audard V, Sartoretti P, Roth AD, Brezault C, Le Charpentier M, Dousset B, Morel P, Soubrane O, Chaussade S, Mentha G and Terris B: Severe hepatic sinusoidal obstruction associated with oxaliplatin-based chemotherapy in patients with metastatic colorectal cancer. Ann Oncol 15: 460-466, 2004.

19 Dindo D, Demartines N and Clavien PA: Classification of surgical complications: a new proposal with evaluation in a cohort of 6336 patients and results of a survey. Ann Surg 240: 205-213, 2004.

20 Rahbari NN, Garden OJ, Padbury R, Brooke-Smith M, Crawford M, Adam R, Koch M, Makuuchi M, Dematteo RP, Christophi C, Banting S, Usatoff V, Nagino M, Maddern G, Hugh TJ, Vauthey JN, Greig P, Rees M, Yokoyama Y, Fan ST, Nimura Y, Figueras J, Capussotti L, Büchler MW and Weitz J: Posthepatectomy liver failure: a definition and grading by the International Study Group of Liver Surgery (ISGLS). Surgery 149: 713-724, 2011.

21 Vauthey JN, Pawlik TM, Ribero D, Wu TT, Zorzi D, Hoff PM, Xiong HQ, Eng C, Lauwers GY, Mino-Kenudson M, Risio M, Muratore A, Capussotti L, Curley SA and Abdalla EK: Chemotherapy regimen predicts steatohepatitis and an increase in 90-day mortality after surgery for hepatic colorectal metastases. J Clin Oncol 24: 2065-2072, 2006.

22 Parikh AA, Gentner B, Wu TT, Curley SA, Ellis LM and Vauthey JN: Perioperative complications in patients undergoing major liver resection with or without neoadjuvant chemotherapy. J Gastrointest Surg 7: 1082-1088, 2003.

23 Delva E, Camus Y, Nordlinger B, Hannoun L, Parc R, Deriaz H, Lienhart A and Huguet C: Vascular occlusions for liver resections. Operative management and tolerance to hepatic ischemia: 142 cases. Ann Surg 209: 211-218, 1989.

24 Nordlinger B, Sorbye H, Glimelius B, Poston GJ, Schlag PM, Rougier P, Bechstein WO, Primrose JN, Walpole ET, FinchJones M, Jaeck D, Mirza D, Parks RW, Mauer M, Tanis E, Van Cutsem E, Scheithauer W and Gruenberger T: Perioperative chemotherapy with folfox 4 and surgery versus surgery alone for resectable liver metastases from colorectal cancer (eortc intergroup trial 40983): a randomised controlled trial. Lancet 371: 1007-1016, 2008.

25 Wang ZM, Chen YY, Chen FF, Wang SY and Xiong B: Perioperative chemotherapy for patients with resectable colorectal hepatic metastasis: a meta-analysis. Eur J Surg Oncol 41: 11971203, 2015. 